\title{
Partial Nephrectomy as Treatment of an Atypical Metastasis from Prostate Cancer-A Case Report and Review of Literature
}

\author{
Ulisses Lopes Guerra Pereira Sobrinho, André Luiz Lima Diniz, \\ Rodrigo Galves Mesquita Martins, Diogo Eugenio Abreu Da Silva, \\ Tomás Accioly De Souza, José Anacleto Dutra De Resende Júnior* \\ Department of Urology, Lagoa Federal Hospital, Rio de Janeiro, Brazil \\ Email: ulisses.sobrinho@yahoo.com.br, andre.ufjf@gmail.com,rodrigogalves2001@hotmail.com, \\ diogoeugenio@hotmail.com, tomasaccioly@gmail.com, ${ }^{*}$ joseanacletojunior@gmail.com
}

How to cite this paper: Sobrinho, U.L.G.P., Diniz, A.L.L., Martins, R.G.M., Da Silva, D.E.A., De Souza, T.A. and De Resende Júnior, J.A.D. (2018) Partial Nephrectomy as Treatment of an Atypical Metastasis from Prostate Cancer-A Case Report and Review of Literature. Open Journal of Urology, 8, 8-16.

https://doi.org/10.4236/oju.2018.81002

Received: November 9, 2017

Accepted: January 16, 2018

Published: January 19, 2018

Copyright $\odot 2018$ by authors and Scientific Research Publishing Inc. This work is licensed under the Creative Commons Attribution International License (CC BY 4.0).

http://creativecommons.org/licenses/by/4.0/

\section{(C) (i) Open Access}

\begin{abstract}
Background: Prostate cancer is the second most common type of cancer in man and the second in cancer-specific deaths in this population in the world. Most of the causes of death related to prostate cancer are due to its distant metastases, with the most common sites being: skeleton, distant lymph nodes, liver and lung. Renal metastasis is rare, and studies suggest infiltration due to arterial microembolization of the tumor. A key point in this scenario is the clinical suspicion of differential diagnoses, to offer the patient an effective therapy in such a specific case. Aim: To report a case of a patient with prostate cancer undergoing partial nephrectomy whose histopathological report revealed a metastatic lesion of that primary site. Case Presentation: 74 years old man, referred in May 2015 due to high PSA level and lumbago. PSA $323.11 \mathrm{ng} / \mathrm{dl}$, rectal examination cT3a; biopsy was performed and histopathological study reported bilateral prostate adenocarcinoma, Gleason's score $8(4+$ 4). Patient's staging showed multiple secondary implants on skeletal scintigraphy. Tomography revealed solid exophytic lesion in the lower pole of the right kidney $(4.7 \times 3.6 \mathrm{~cm})$; prostate without cleavage planes with seminal vesicles and pelvic node enlargement. Hormone therapy was initiated, PSA levels dropped to $9.51 \mathrm{ng} / \mathrm{dl}$ and total testosterone $<50 \mathrm{ng} / \mathrm{dl}$. Partial nephrectomy was planned, initially by laparoscopy, but converted to laparotomy in December 2015. Procedure lasting 3 hours, minimal blood loss, no perioperative complications, discharged on the 3rd postoperative day. Histopathological report described undifferentiated malignant neoplasm, requiring immunohistochemistry that confirmed prostate adenocarcinoma. Patient remains hormone therapy, with no progression of the disease so far. Conclusion:
\end{abstract}


Given the rarity of these cases, it is not possible to presume that nephrectomy enhances the survival rates. However, we observed that partial nephrectomy was a good choice for our patient, being the first case described in the literature. More reports should be available and studies with higher levels of evidence should be conducted to assist us in patient orientation and decision making.

\section{Keywords}

Advanced Prostate Cancer, Kidney Metastasis, Partial Nephrectomy

\section{Introduction}

Prostate cancer (PC) still remains the most frequent oncologic disease in the urogenital tract, the second most frequently diagnosed cancer among men and the fifth leading cause of cancer death worldwide [1].

Screening may increase chances of disease detection in early stages, however, despite all debates about this matter, PC is often discovered in advanced stages in about to $17 \%$ of patients, in which the risk of cancer-specific mortality is increased [2].

The common routes of metastatic spread from the prostate are through veins from prostate to the paravertebral or Batson's plexus, via lymphatic system, arterial, and by direct extension. Metastatic spread is usually associated with skeletal, pulmonary or hepatic masses and more locally invades the seminal vesicles, the bladder, the rectum and regional lymph nodes [3].

Literature has shown diverse sites of possible metastases, these atypical metastatic locations being the ocular region, brain, respiratory tract, testes, mammary and parotid glands, the skin and the lymphatic system [4]. Some studies reported that $15 \%$ of men with PC might be affected by atypical metastases at diagnosis [5] [6].

Metastasis to the kidney is infrequent and its management is controversial from diagnosis to treatment, as a few publications are available.

The aim of this paper is to report a case of a patient with PC undergoing partial nephrectomy whose histopathological report revealed a metastatic lesion of that primary site. To the best of our knowledge it is the first case described in literature.

\section{Case Presentation}

A 74-year-old male with a history of low back pain went to our hospital for clinical solution. In May 2015, present increased serum prostate specific antigen (PSA) of $323.11 \mathrm{ng} / \mathrm{dl}$; cT3a on rectal digital examination. A prostate biopsy was performed and histopathological report was a bilateral Gleason $8(4+4)$ adenocarcinoma. Bone scan and computed tomography (CT) was obtained. Multiple secondary bone implants was identified in bone scan. CT revealed: solid exo- 
phytic lesion in the lower pole of the right kidney, $4.1 \times 3.6 \mathrm{~cm}$ (Figure 1), with soft tissue density, impregnated by contrast; prostate had no cleavage planes to the seminal vesicles, no retroperitoneal lymph node enlargement, but lymphadenopathy at obturator nodes bilaterally and iliac nodes on the right. Complete hormonal blockade was initiated. The first PSA after hormonal deprivation was 9.51 $\mathrm{ng} / \mathrm{dl}$ and total testosterone $<50 \mathrm{ng} / \mathrm{dl}$.

In view of all these results, partial nephrectomy was proposed and the surgery was initiated by laparoscopic approach, but converted to laparotomy access. Procedure lasted 3 hours with minimal blood loss, discharged on 3rd postoperative day.

Patient returned after 15 days to postoperative review with no pain or complications in surgery site. Histopathological report described undifferentiated malignant neoplasm, immunohistochemistry with PSA markers was found in cells, corresponding to prostate adenocarcinoma (Figure 2).

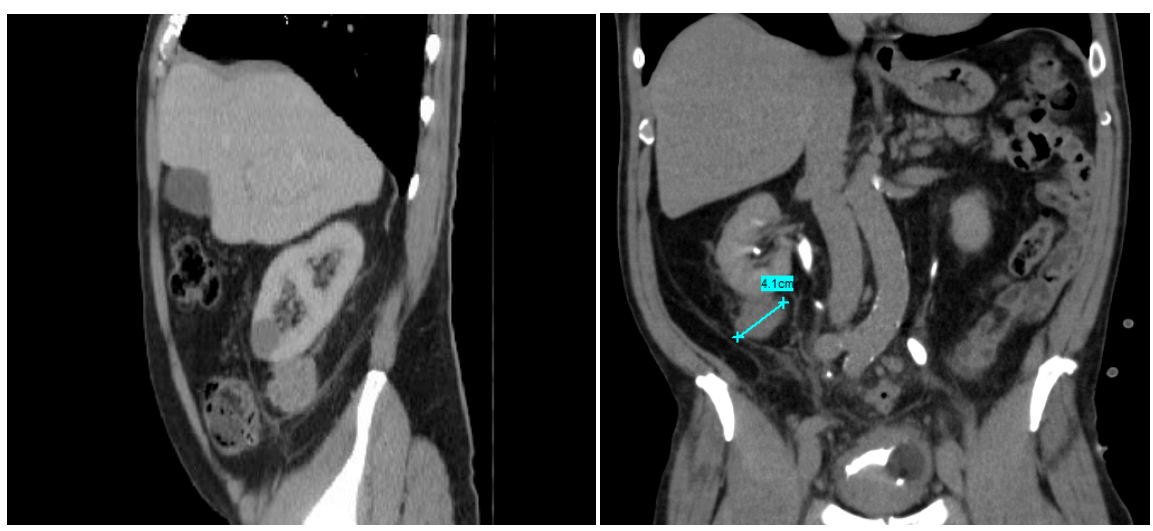

Figure 1. CT presenting solid exophytic lesion in the lower pole of the right kidney, $4.1 \times$ $3.6 \mathrm{~cm}$ with soft tissue density.

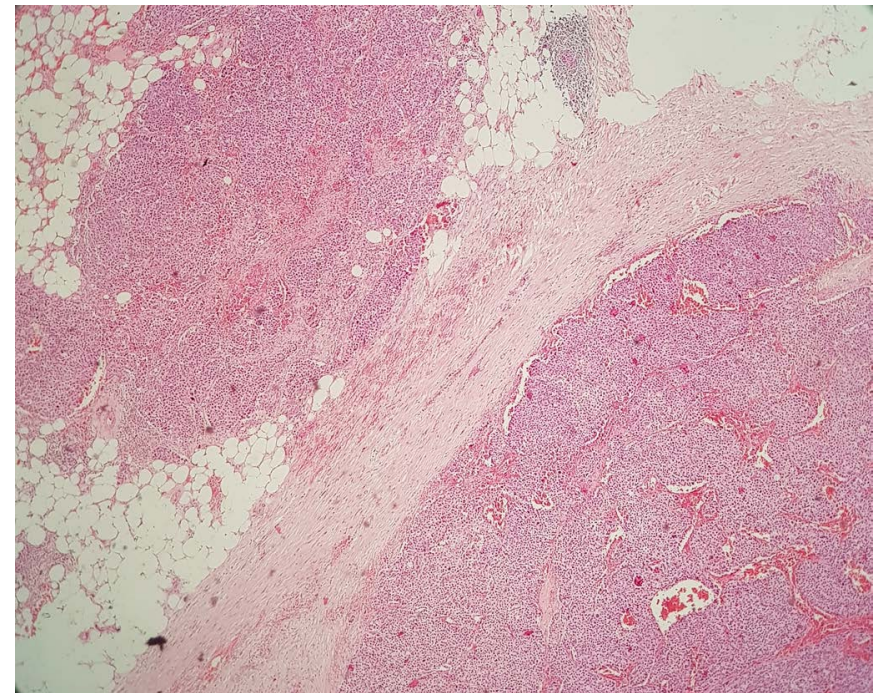

Figure 2. Histopathological images show undifferentiated malignant neoplasm that was identified as prostate adenocarcinoma in immunohistochemistry by positive PSA. 
After 10 months of partial nephrectomy, the patient had disseminated bone pain, with difficulty to walk. This evolution of pain was in the presence of hormonal blockade, with progressive increase of PSA. New bone scan was performed, which identified an increase in disseminated osteogenic activity, mainly in the left femur, where the patient experienced more pain. X-ray of the left femur confirmed pathological fracture, which was followed up by the orthopedic team.

Chemotherapy for disease control was started. Patient still under treatment, but now with significant renal impairment and general condition, hospitalized for improvement of the health.

\section{Discussion}

Despite the great discussion within the scientific community, PC screening through PSA has led to intriguing scenarios. A Cochrane review suggests that PSA screening is associated with an increased diagnosis rate, the detection of more localized and less advanced disease (T3-4, N1, M1), but overall survival and cancer-specific survival had no benefit observed [7].

From overdiagnosis to overtreatment, the debate has never ignored the fact that patient's participation is substantial, and that all information should serve as substrate for patient's and team's decision making [8] [9].

Scosyrev and colleagues carried out a study on PC identified in elderly population; they observed frequency of M1 PC at presentation was 3\% for the group aged $<75$ years, $5 \%$ for the group ages 75 to 79 years, $8 \%$ for the group ages 80 to 84 years, $13 \%$ for the group ages 85 to 89 years, and $17 \%$ for the group aged 90 years. In their analyses, data indicates that, compared with younger patients (aged $<75$ years), older patients were more likely to present with very advanced disease and contributed almost half (48\%) of all M1 cases [2].

Gandaglia et al. analysed data from US Healthcare Cost and Utilization Project Nationwide Inpatient Sample between 1998 and 2010. About 74,826 patients with metastatic PC were identified. The most common metastatic sites were bone (84\%), distant lymph nodes (10.6\%), liver (10.2\%), and thorax (9.1\%). Kidney/adrenal metastasis was observed in 757 individuals. The distribution of metastatic sites in patients with a single site involved was $0.3 \%$ for those with kidney/adrenal involvement; and in those with two or more sites it becomes $4.2 \%$.

$\mathrm{Wu}$ et al. reviewed their intra-institutional cases of metastasis to the kidney; according to the survey, 43 cases were identified; only one case had a correlation with PC [10].

As usually, challenging cases are brought to discussion rounds at scientific meetings of our service. Some differential diagnosis was discussed; a second primary tumor was possible, a tumor-to-tumor metastasis was carried out and the prostate to kidney metastasis was also considered.

In ages before PSA or CT, one journal published two articles reporting tumor-to-tumor metasis. In one paper Rabson et al. reported two cases; first a 
72-year-old man which harbored a clinically silent PC with spread to an equally silent clear-cell carcinoma of the right kidney; the second one presented a 56-year-old man who also manifested spread of PC to various organs, including the right kidney that contained a renal cell carcinoma (RCC) [11]. In the other paper, Schneider described a 60-year-old man who suffered low urinary tract symptoms for six months progressed to haematuria; an enlarged and fixed prostate was observed on physical examination, cystotomy was performed and biopsy revealed adenocarcinoma; patient died 2 weeks later. Necropsy identified a left kidney mass, which histopathological study demonstrated RCC and PC in the same tissue [12].

In 1996, Inatomi et al. published a similar case which indicates in 1985, either before PSA screening; in this case, a 62-year-old-man was diagnosed with metastatic PC, received hormonal blockade; three years later found an image on CT suggesting RCC. It was supposed to be a second primary tumor; after follow up, death came in 1990. Necropsy was performed, demonstrated metastases in regional and para-aortic lymph nodes, lungs, left adrenal gland, pleurae, ribs, vertebrae and pelvic bone; left kidney was taken to histopathology study, observing PC in the same tissue as RCC [13].

More recently two other papers have been published about tumor-to-tumor metastasis; Horn et al. described a case of a kidney resected for a primary neoplasm (oncocytoma) that harbored metastases from a clinically undiagnosed PC in a 92-year-old man; Desai et al. reported a 72-year-old man who found a kidney mass during the staging of his newly discovered PC; after nephrectomy, histopathological found typical RCC along with extensive involvement by PC [14].

Due to the small incidence of prostatic tumor leading to renal metastasis, differential diagnosis must be thought like a second primary RCC and benign lesions such as angiomyolipoma or oncocytoma. Some groups have done biopsy before treating those masses. Kutcher et al. described the first pre-mortem diagnosis of prostatic metastasis to kidney; since then cases like these have been scarcely reported in literature [13]. Ibinaiye et al. described the second case of renal metastasis from prostatic adenocarcinoma diagnosed antemortem by percutaneous fine needle aspiration in literature [14]. Chan et al. reported a biochemical recurrence of an androgen-refractory metastatic PC, which staging found an enormous mass in the right kidney; biopsy was taken before hard decisions were made [15]. Chen et al. described a diffuse renal and retroperitoneal mass observed in CT, in which ultrasonography-guided biopsy confirmed the prostate metastatic kidney disease [16].

One of the hardest situations described came from Alaini et al., who identified a prostate metastasis into a renal allograft. A 76-year-old man with a history of end-stage related to IgA nephropathy, was transplanted, and found PC two years after receiving his new organ, submitted to radiotherapy as first line treatment. Three years later, he was admitted into the hospital with pulmonary conditions and renal impairment. The tomographic findings around the allograft kidney 
were essential in decision making and a biopsy was performed. It identified amyloid deposition and significant interstitial fibrosis with tubular atrophy. There was no evidence of rejection. However, up to $50 \%$ of the renal parenchyma was involved by adenocarcinoma. Diagnosis of metastatic prostate carcinoma with infiltration of the allograft, retroperitoneal, and pelvic nodes were made. Facing this diagnosis, patient opted for palliative care. Dialysis was discontinued, and he died at home [17].

Gunlusoy et al. and Denti et al. respectively reported postmortem findings of bilateral and unilateral renal metastases from PC which was initially misdiagnosed as RCC [18] [19]. Sakata et al. reported a 67-year-old man found to have a renal mass on CT incidentally. He had had total androgen blockade and chemotherapy for treatment of PC discovered 33 months before the incidental finding. Based on clinical features and radiologic results, it was thought to have a second malignant tumor; left nephrectomy was performed. The pathological finding of this case was renal metastasis from prostatic adenocarcinoma. Patient died 18 months post-nephrectomy [20].

The most usual clinical presentation reported was lumbar pain, but some cases are shown in different manners. Gallego et al. published a patient presented with acute renal failure secondary to metastasis from PC to the kidneys [21]. Gunlusoy et al., reported a case which symptoms were left flank pain, hematuria and vomiting for one month [18]. Ibinaiye et al. presented an infected renal cyst as a result of metastatic prostatic adenocarcinoma with widespread bone metastases [14].

As shown, the incidence of renal metastasis from adenocarcinoma of the prostate is low, demonstrated in the literature only by some case reports. It is a major diagnostic challenge, due to the risk of a second malignant neoplasm. It is necessary to have the clinical suspicion of the possibility of being a metastasis, even if it is atypical.

In view of this, this article becomes necessary for the knowledge of one of the forms of presentation of the disease, aiding specialists in new future cases.

The summary of literature is found in Table 1.

\section{Conclusions}

The data about PC is far from exhausted. Our current knowledge has not yet reached the limits of what is necessary to understand the singularities of this disease that has pushed us beyond our barriers.

Once a renal lesion is found in the context of PC, there is a possibility of facing a metastatic lesion. Even with small chances of being prostatic metastasis to the kidney, a biopsy should be considered, so the next steps will be well defined. A few options for the treatment of metastatic renal masses from primary prostate tumor are available such as nephrectomy, chemotherapy and radiotherapy, and renal artery embolization.

Given the rarity of these cases, it is not possible to presume that nephrectomy 
Table 1. Summary of literature.

\begin{tabular}{|c|c|c|c|c|c|c|c|}
\hline Year & Author & PSA & LNM & Bone Mx & Histopathological Assessment & Nephrectomy & Disclosure \\
\hline 1954 & Rabson [11] & - & - & - & Necropsy & No & - \\
\hline 1955 & Schneider [12] & - & Yes & Yes & Necropsy & No & - \\
\hline 1986 & Kutcher [13] & - & Yes & No & Ultrasound-guided biopsy & No & Death in 3 months \\
\hline 1996 & Inatomi $[22]$ & - & No & Yes & Necropsy & No & Death in 24 months \\
\hline 1996 & Gallego [21] & 800 & Yes & Yes & Laparotomic biopsy & No & Unknown \\
\hline 1998 & Denti [19] & 82.3 & Yes & No & Nephrectomy & Yes & Death in 5 months \\
\hline 2004 & Gunlusoi [18] & 150 & Yes & Yes & Laparotomic biopsy & No & Death in 12 months \\
\hline 2011 & Sakata $[20]$ & 21.2 & Yes & Yes & Nephrectomy & Yes & Death in 18 months \\
\hline 2012 & Ibinaiye [14] & 150 & - & Yes & Ultrasound-guided biopsy & - & Patient quit treatment \\
\hline 2012 & Horn [23] & 500 & - & - & Nephrectomy & Yes & - \\
\hline 2013 & Desai [24] & 19 & Yes & Yes & Nephrectomy & Yes & Death in 1 month \\
\hline 2013 & Chan [15] & 200 & Yes & No & CT guided biopsy & No & Death \\
\hline 2016 & Chen [16] & 36.8 & Yes & No & Ultrasound-guided biopsy & No & Patient quit treatment \\
\hline 2017 & Alaini [17] & 10 & Yes & No & Ultrasound-guided biopsy & No & Patient quit treatment \\
\hline 2017 & Our case & 32,311 & Yes & Yes & Partial Nephrectomy & No & Patient is on follow up \\
\hline
\end{tabular}

PSA-Prostatic-Specific-Antigen; LNM-Lymph Nodes Metastasis; Bone Mx-Bone Metastasis.

enhances the survival rates. However, we observed that partial nephrectomy was a good choice for our patient, being the first case described in the literature.

More reports should be available and studies with higher levels of evidence should be conducted to assist us in patient orientation and decision making.

\section{References}

[1] Mottet, N., Bellmunt, J., Bolla, M., Briers, E., Cumberbatch, M.G., De Santis, M., et al. (2017) EAU-ESTRO-SIOG Guidelines on Prostate Cancer. Part 1: Screening, Diagnosis, and Local Treatment with Curative Intent. European Urology, 71, 618-629. https://doi.org/10.1016/j.eururo.2016.08.003

[2] Scosyrev, E., Messing, E.M., Mohile, S., Golijanin, D. and Wu, G. (2012) Prostate Cancer in the Elderly: Frequency of Advanced Disease at Presentation and Disease-Specific Mortality. Cancer, 118, 3062-3070. https://doi.org/10.1002/cncr.26392

[3] Wein, A.J., Kavoussi, L.R., Novick, A.C., Partin, A.W. and Peters, C.A. (2011) Campbell-Walsh Urology: Expert Consult Premium Edition: Enhanced Online Features and Print, 4-Volume Set. Elsevier Health Sciences, Philadelphia, 2729 p.

[4] Khan, F., Mahmalji, W., Sriprasad, S. and Madaan, S. (2013) Prostate Cancer with Metastases to the Kidney: A Rare Manifestation of a Common Disease. BMJ Case Reports, 2013, Article ID: bcr2012008388. https://doi.org/10.1136/bcr-2012-008388

[5] Vinjamoori, A.H., Jagannathan, J.P., Shinagare, A.B., Taplin, M.-E., Oh, W.K., Van den Abbeele, A.D., et al. (2012) Atypical Metastases from Prostate Cancer: 10-Year Experience at a Single Institution. AJR American Journal of Roentgenology, 199, 367-372. https://doi.org/10.2214/AJR.11.7533

[6] Long, M.A. and Husband, J.E. (1999) Features of Unusual Metastases from Prostate Cancer. The British Journal of Radiology, 72, 933-941.

https://doi.org/10.1259/bjr.72.862.10673942 
[7] Hayes, J.H. and Barry, M.J. (2014) Screening for Prostate Cancer with the Prostate-Specific Antigen Test: A Review of Current Evidence. JAMA, 311, 1143-1149. https://doi.org/10.1001/jama.2014.2085

[8] Moyer, V.A. and US Preventive Services Task Force (2012) Screening for Prostate Cancer: US Preventive Services Task Force Recommendation Statement. Annals of Internal Medicine, 157, 120-134. https://doi.org/10.7326/0003-4819-157-2-201207170-00459

[9] Bibbins-Domingo, K., Grossman, D.C. and Curry, S.J. (2017) The US Preventive Services Task Force 2017 Draft Recommendation Statement on Screening for Prostate Cancer: An Invitation to Review and Comment. JAMA, 317, 1949-1950. https://doi.org/10.1001/jama.2017.4413

[10] Wu, A.J., Mehra, R., Hafez, K., Wolf, J.S. and Kunju, L.P. (2015) Metastases to the Kidney: A Clinicopathological Study of 43 Cases with an Emphasis on Deceptive Features. Histopathology, 66, 587-597. https://doi.org/10.1111/his.12524

[11] Rabson, S.M., Stier, P.L., Baumgartner, J.C. and Rosenbaum, D. (1954) Metastasis of Cancer to Cancer. American Journal of Clinical Pathology, 24, 572-579. https://doi.org/10.1093/ajcp/24.5.572

[12] Schneider, L.A. (1955) Metastasis of Cancer to Cancer; Report of a Case. American Journal of Clinical Pathology, 25, 1288-1289. https://doi.org/10.1093/ajcp/25.11.1288

[13] Kutcher, R., Greenebaum, E., Rosenblatt, R. and Moussouris, H.F. (1986) Prostatic Carcinoma Metastasis to the Kidney: Diagnosis by Thin Needle Aspiration Biopsy. Urologic Radiology, 8, 98-100. https://doi.org/10.1007/BF02924087

[14] Ibinaiye, P.O., Mbibu, H., Shehu, S.M., David, S.O. and Samaila, M.O. (2012) Renal Metastasis from Prostate Adenocarcinoma: A Potential Diagnostic Pitfall. Annals of African Medicine, 11, 230-233. https://doi.org/10.4103/1596-3519.102854

[15] Chan, S.W., Ramaseshan, A., Alexiev, B. and Phelan, M. (2013) Late Recurrence of Prostate Cancer Presenting as a Renal Mass. American Journal of Cancer Case Reports, 1, 35-38.

[16] Chen, C., He, H., Yu, Z., Qiu, Y. and Wang, X. (2016) Renal and Retroperitoneal Metastasis from Prostate Adenocarcinoma: A Case Report. World Journal of Surgical Oncology, 14, 74. https://doi.org/10.1186/s12957-016-0834-4

[17] Alaini, A., Singh, P., Shah, R., Fischer, E., Ganta, K., Barrett, T., et al. (2017) Prostate Cancer Metastatic to the Renal Allograft: A Case Report. Transplantation Proceedings, 49, 201-205. https://doi.org/10.1016/j.transproceed.2016.11.031

[18] Gunlusoy, B., Arslan, M., Selek, E., Sayhan, H.S., Minareci, S. and Cicek, S. (2004) A Case Report: Renal Metastasis of Prostate Cancer. International Urology and Nephrology, 36, 555-557. https://doi.org/10.1007/s11255-004-0850-0

[19] Denti, F., Wisard, M., Guillou, L., Francke, M.L. and Leisinger, H.J. (1999) Renal Metastasis from Prostatic Adenocarcinoma: A Potential Diagnostic Pitfall. Urologia Internationalis, 62, 171-173. https://doi.org/10.1159/000030384

[20] Sakata, R., Iwasaki, A., Kobayashi, M., Osaka, K., Fujikawa, A., Tsuchiya, F., et al. (2011) Renal Metastasis from Prostatic Adenocarcinoma: A Case Report. Hinyokika Kiyo, 57, 683-687.

[21] Galindo Gallego, M., Diest Perez, A., Lopez Elzaurdia, C., Fernández Aceñero, M.J. and Manzanares Sacristán, J.J. (1996) Atypical Metastasis from Prostatic Carcinoma. Report of a Case of Renal Metastasis and Literature Review. Minerva Urologica e Nefrologica, 48, 183-187. 
[22] Inatomi, H., Yamada, Y. and Okamura, T. (1996) A Case of Prostate Carcinoma Metastasizing to Renal Cell Carcinoma. International Journal of Urology, 3, 155-157. https://doi.org/10.1111/j.1442-2042.1996.tb00503.x

[23] Horn, A.J., Fritz, B.E., Lagrange, C.A., West, W.W. and Lele, S.M. (2012) Clinically Undiagnosed Prostate Carcinoma Metastatic to Renal Oncocytoma. Case Reports in Urology, 2012, Article ID: 307813.

[24] Vyas, M., Menon, S. and Desai, S.B. (2013) Collision Tumor of Kidney: A Case of Renal Cell Carcinoma with Metastases of Prostatic Adenocarcinoma. Indian Journal of Medical and Paediatric Oncology, 34, 21-23.

https://doi.org/10.4103/0971-5851.113409

\section{List of Abbreviations}

PSA: Prostatic-Specific-Antigen

CT: Computerized Tomography

PC: Prostate Cancer

RCC: Renal Cell Carcinoma 